\title{
IGFBP-2 in cervical cancer development
}

\author{
Gurjeet Kaur*, Shandra Devi Balasubramaniam, Yung Jen Lee \\ Institute for Research in Molecular Medicine, Universiti Sains Malaysia, 11800 Minden, Pulau Pinang, Malaysia
}

\section{A R T I C L E I N F O}

\section{Keywords:}

Cervical cancer

Cervical intraepithelial neoplasia

IGFBP-2

IGF-1

\begin{abstract}
A B S T R A C T
Objective: Increased expression of insulin-like growth factor binding protein 2, IGFBP-2, is associated with many cancers, though its role in cervical cancer is unclear. The aim of this study was to investigate the expression of IGFBP-2 protein and the transcriptomics profile of genes involved in the IGF signaling pathway during cervical cancer development.

Design: Immunohistochemical expression of IGFBP-2 protein was semi-quantitatively assessed in tissue microarrays containing 9 normal cervix, 10 low grade cervical intraepithelial neoplasia (LGCIN), 10 high grade cervical intraepithelial neoplasia (HGCIN) and 42 squamous cell carcinoma (SCC) cases. The gene expression profiles of IGFBP-2, IGF-1, IGF-1R, PTEN, MDM2, AKT1 and TP53 were determined in three cervical tissue samples each from normal cervix, human papillomavirus (HPV)-infected LGCIN, HGCIN and SCC, using Human Transcriptome Array 2.0.

Results: IGFBP-2 protein was highly expressed in the cytoplasm of SCC cells compared to normal cervix $(p=.013)$. The expression was not significantly associated with CIN grade or SCC stage. Transcriptomics profiling demonstrated upregulation of IGFBP-2 and TP53 in HGCIN and SCC compared to normal cervix. IGF-1, IGF-1R and PTEN genes were downregulated in all histological groups. IGF-1 gene was significantly downregulated in SCC $(p=.031)$, while PTEN gene was significantly downregulated in HGCIN $(p=.012)$, compared to normal cervix. MDM2 and AKT1 genes were downregulated in LGCIN and HGCIN, while upregulated in SCC. Conclusion: In cervical carcinogenesis, IGFBP-2 appears to play an oncogenic role, probably through an IGFindependent mechanism.
\end{abstract}

\section{Introduction}

The IGF binding proteins (IGFBPs) comprise a family of six proteins, IGFBP1-6, which bind the two peptide ligands of insulin-like growth factors 1 and 2 (IGF-1 and IGF-2) (Firth and Baxter, 2002). The six IGFBPs have distinct functional properties. IGFBP-2 is produced in the liver, and is expressed in many normal tissues including male and female reproductive tracts, gastrointestinal tissues, liver, gallbladder, and other tissues (Uhlen et al., 2010). IGFBP-2 exerts its cellular effects through two main mechanisms; the IGFBP-2-IGF-dependent pathway is related to its interaction with IGF-1 and IGF-2 for ligand-binding, as well as components of the extracellular matrix and cell surface proteoglycans. Whereas, the IGF-independent actions are exerted when IGFBP-2 interacts with integrin receptors or when it induces specific target genes in the nucleus (Hoeflich et al., 2001; Pollak et al., 2004; Rosenzweig, 2004; Russo et al., 2015; Weroha and Haluska, 2012; Yau et al., 2015). The role of IGFBP-2 in tumorigenesis is complex and evidence is contradictory for the reason that it can act either in a tumor suppressor or oncogenic manner. IGFBP-2 has been generally shown to inhibit IGF actions, hence inhibit mitogenesis, differentiation, survival, and other cellular processes (Russo et al., 2015). This is often attributed to the ability of IGFBP-2 to compete with IGF for IGF receptors (IGFR) binding. However, there is also evidence of IGFBP-2 exerting oncogenic properties that promote cell proliferation, invasion and suppress apoptosis, through interaction with integrins (Hoeflich et al., 2001; Pickard and McCance, 2015; Weroha and Haluska, 2012; Yau et al., 2015). IGFBP-2 has also been shown to correlate with metastasis, using integrin-dependent mechanisms to reduce cell adhesion, and promote invasion (Schutt et al., 2004).

Elevated IGFBP-2 serum levels have been reported in various cancers (Weroha and Haluska, 2012) including lung (Guo et al., 2013), colon (Liou et al., 2010; Renehan et al., 2000), prostate (Ho and Baxter,

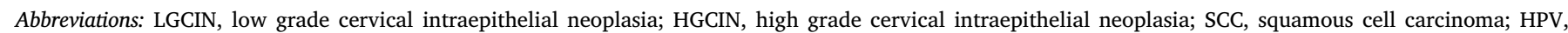

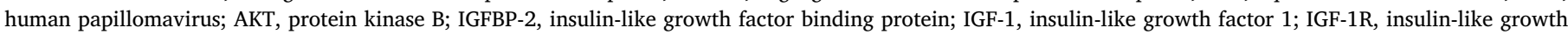

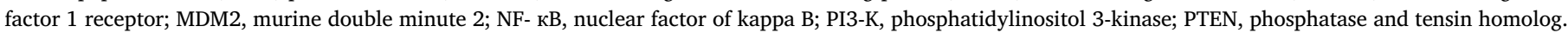

* Corresponding author.

E-mail address: gurjeet@usm.my (G. Kaur).
} 
1997), ovary (Flyvbjerg et al., 1997), and is often associated with advanced stage and poor prognosis (Elmlinger et al., 2001; Fukushima and Kataoka, 2007; Guo et al., 2013; Liou et al., 2010; Sayer et al., 2005). Though serum levels of IGFBP-2 were found to increase with squamous cell cervical cancer stage (Zhi et al., 2014), its expression in the tumor microenvironment is unclear. In tissues, IGFBP-2 protein was over-expressed in invasive prostatic cancer (Ambrosini-Spaltro et al., 2011), hepatocellular carcinoma (Zhou et al., 2012), high grade gliomas and glioblastoma multiforme (Elmlinger et al., 2001; Fukushima and Kataoka, 2007; Jiang et al., 2006). On the contrary, few studies reported low or absent immunohistochemical expression of IGFBP-2 in specific histologic type of cancers, such as clear cell and mucinous ovarian carcinomas (Wang et al., 2006), as well as adenocarcinoma and squamous cell carcinoma of the lung (Yazawa et al., 2009).

Besides regulation of IGF levels in the circulation, and interactions with extracellular matrix and integrin receptors, IGFBP-2 also exerts influence on the proteins within the cell cytoplasm and target genes in the nucleus via the IGF-dependent pathway (Russo et al., 2015). After ligand binding with IGF receptors, signals will stimulate intracellular downstream networks, one of which is the phosphatidylinositol 3-kinase - protein kinase B (PI3K-AKT) system which regulates cell growth, proliferation, survival, migration and cell invasion (Janku et al., 2018; Tang et al., 2018; Vivanco and Sawyers, 2002). Dysregulation of the AKT pathway is linked to several types of cancers (Tang et al., 2018; Vivanco and Sawyers, 2002). Phosphatase and tensin homolog (PTEN) is an antagonist to the PI3K-AKT system. Mutations or silencing of the PTEN tumor suppressor gene can lead to PI3K-signaling deregulation and reduction of the PTEN enzyme, which eventually causes uncontrolled cell growth and cancer formation (Li et al., 1997; Vivanco and Sawyers, 2002). PTEN expression has an inverse association with IGFBP-2 expression in breast cancer and glioma (Dean et al., 2014; Fukushima and Kataoka, 2007; Levitt et al., 2005; Perks et al., 2007). AKT may influence cell survival indirectly through phosphorylation and activation of nuclear factor of kappa B (NF- кB) that mediates nuclear translocation and inhibits apoptotic genes (Romashkova and Makarov, 1999; Vivanco and Sawyers, 2002). AKT can also regulate the p53 tumor suppressor gene through phosphorylation of p53-binding protein (Vivanco and Sawyers, 2002). The cellular oncoprotein murine double minute 2 (MDM2) and p53 are linked through a negative feedback loop in which p53 growth suppressive function is limited in unstressed cells (Moll and Petrenko, 2003; Nag et al., 2013). TP53 tumor suppressor gene is inactivated in cancers through several mechanisms, including p53 protein association with viral proteins and high levels of MDM2 (Momand et al., 1998). MDM2 is also regulated through phosphorylation by AKT, which allows efficient translocation of MDM2 into the nucleus where it can bind to p53, resulting in enhanced p53 degradation (Moll and Petrenko, 2003; Vivanco and Sawyers, 2002). p53 positively regulates the PTEN promoter, thus inhibiting the PI3K-AKT activity. In summary, IGFBP-2 can cause cellular effects in an IGF-dependent or IGF-independent manner which triggers downstream cellular mechanisms that are potentially oncogenic.

The transformation of normal cervical epithelial cells into a distinctive pre-cancerous stage or cervical intraepithelial neoplasia (CIN), and further into squamous cell carcinoma (SCC) offers a unique advantage to study the role of IGFBP-2 in cervical carcinogenesis. Our aim was to investigate the expression of IGFBP-2 protein in the various stages of cervical cancer development, and to determine the gene expression profiles involved in the IGF signaling pathway.

\section{Material and methods} 2.1. Evaluation of immunohistochemical expression of IGFBP-2 protein in
tissue microarrays

Two tissue microarrays (TMA) (BB10011 and CR1003, Biomax USA) containing 10 cases of normal cervix, 10 low grade cervical intraepithelial neoplasia (LGCIN), 10 high grade cervical intraepithelial neoplasia (HGCIN) and 43 squamous cell carcinoma (SCC) cases, with two identical tissue cores per case, were immunohistochemically stained for IGFBP-2 protein. The TMAs were accompanied with the pathology diagnosis, CIN grade (Bethesda Classification System) and FIGO staging for SCC cases.

Briefly, after deparaffinization and rehydration, antigen retrieval was performed by soaking the slides in citrate buffer ( $10 \mathrm{mM}, \mathrm{pH} 6.0)$ and heated in a microwave oven on low setting for $20 \mathrm{~min}$. After treatment with peroxidase-blocking solution (Dako, USA), sections were blocked with $5 \%$ bovine serum albumin (Sigma-Aldrich, USA) and $5 \%$ goat serum (Biowest, France) in tris-buffered saline Tween-20 (TBS$\mathrm{T})$ solution at room temperature for $1 \mathrm{~h}$. Primary rabbit monoclonal anti-IGFBP2 antibody [EPR3380(2), Abcam] 1:100 dilution was applied and incubated overnight at $4{ }^{\circ} \mathrm{C}$. Then, biotinylated secondary antibody goat anti-rabbit IgG H\&L (Biotin), (Code No. AB6720, Abcam) was added for $1 \mathrm{~h}$, followed by incubation with avidin-biotin complex (R.T.U Vectastain ABC reagent, Vector Laboratories, USA) for $1 \mathrm{~h}$. Slides were rinsed thrice with TBS-T between the incubation steps. DAB solution (3,3'-diaminobenzidine in chromogen solution, Dako, USA) was applied for $10 \mathrm{~min}$, then rinsed and counterstained with Mayer's haematoxylin (Dako, USA). The slides were dried, mounted and viewed under a light microscope before scanned using Hamamatsu, NanoZoomer S60 Digital Slide Scanner. Brown staining signified positive protein expression. The histoscore was calculated by multiplying the percentage of positivity score and staining intensity score, as shown in Table 1. As each case was represented by two cores, an average histoscore was taken. Endometrial carcinoma was used as positive control, while for negative control, primary antibody was not added.

\subsection{Tissue samples, RNA extraction, Human Transcriptome Array 2.0}

Formalin-fixed paraffin-embedded tissues of histologically confirmed normal cervix, low grade cervical intraepithelial neoplasia or CIN 1 (LGCIN), high grade cervical intraepithelial neoplasia comprising CIN 2 and CIN 3 (HGCIN) and squamous cell carcinoma (SCC) were retrieved from pathology archives. This part of the study was approved by the Human Research Ethics Committee of Universiti Sains Malaysia. Only HPV 16 and/or 18 positive CIN and SCC samples identified by immunohistochemistry using p16INK4a and HPV16 E6 + HPV18 E6 antibodies, and real time-PCR were included. Altogether there were 12 samples, comprising three samples each of normal cervix as control (HPV negative), HPV-positive LGCIN, HGCIN and SCC. Laser capture microdissection was used to dissect the area of interest. Total RNA was extracted from the cervical epithelium using RNeasy FFPE extraction kit (Qiagen, Germany). The RNA purity was measured using Nanodrop and quantified using a bioanalyzer (Agilent, USA).

GeneChip Human Transcriptome Array 2.0 (HTA 2.0) Affymetrix, USA was used to profile the gene signatures in the different histological groups. Sensation plus FFPE WT kit (Affymetrix, USA) was used

\section{Table 1}

Histoscore method for assessing immunohistochemical expression of IGFBP-2 protein.

\begin{tabular}{llll}
\hline Percentage of positivity & Score & Staining intensity & Score \\
\hline$<1 \%$ positive cells & 0 & & \\
$1 \%-25 \%$ positive cells & 1 & No staining & 0 \\
$26 \%-50 \%$ positive cells & 2 & Weak staining & 1 \\
$51 \%-75 \%$ positive cells & 3 & Moderate staining & 2 \\
$\geq 75 \%$ positive cells & 4 & Strong staining & 3 \\
\hline
\end{tabular}

Final histoscore $=$ percent positivity score $\mathrm{X}$ staining intensity score

Score: $0=$ Negative

$1-3 / 12=$ Low expression

$\geq 4 / 12=$ High expression 
according to the manufacturer's protocol. Upon hybridization, the chip was scanned using Affymetrix GeneChip Scanner 3000. The data was analyzed by Affymetrix GeneChip Operating Software (GCOS) which contains qualitative and quantitative analysis for every probe set. Affymetrix ${ }^{\circledast}$ Transcriptome Analysis Console (TAC) software was used to determine the IGFBP-2, IGF-1, IGF-1R, PTEN, MDM2 and AKT1 transcriptomic profiles in each histological group compared to normal cervix.

\subsection{Statistical analysis}

The association between IGFBP-2 protein expression and histological groups as well as clinicopathological parameters were analyzed by the chi-square test with statistical significance set at $p$ value $<.05$, using IBM SPSS V24.0 software package for Windows. Transcriptomic analysis in HTA 2.0 was performed by comparing gene expression in each histological group to normal cervix, using one-way ANOVA test provided by the array software, with significance set at $p$ value $<.05$.

\section{Results}

IGFBP-2 immunohistochemical expression was scored on 71 cases comprising 9 normal cervix, 10 LGCIN, 10 HGCIN and 42 SCC cases. One case of normal cervix was devoid of squamous epithelium and one SCC tissue was detached, hence excluded from the analysis. IGFBP-2 protein was localised in the cytoplasm and membrane of cervical squamous epithelial cells. In the normal cervix, squamous epithelial cells showed weak staining in suprabasal cells only. In CIN, basal cells and superficial epithelial cells showed positive staining, while majority of neoplastic cells in SCC were positive. Stromal inflammatory cells were positively stained. IGFBP-2 protein was ubiquitously expressed in 68 of 71 cases $(95.8 \%)$. The histoscore was significantly higher in SCC compared to normal cervix $(p=.002)$. The histoscores are tabulated in Table 2 and IGFBP-2 immunohistochemical staining patterns are shown in Fig. 1. There was no significant association between IGFBP-2 expression with CIN grade $(p=.638)$ or SCC FIGO Staging System $(p=.441)$.

Transcriptomic profiles of IGFBP-2, IGF-1, IGF-1R, PTEN, MDM2 and AKT1 genes were compared between each histological group and normal cervix. The differences in fold change are shown in Fig. 2. The results demonstrated IGFBP-2 gene downregulation in LGCIN, while upregulated in HGCIN and SCC groups, compared to normal cervix. IGF-1, IGF-1R and PTEN genes were increasingly downregulated in the progression from LGCIN to SCC. Both MDM2 and AKT1 gene expressions were downregulated in LGCIN and HGCIN while upregulated in SCC. TP53 gene showed upregulation in HGCIN and SCC.

Statistical analysis demonstrated a significant downregulation of IGF-1 gene expression in SCC compared to normal cervix $(p=.031)$, while PTEN gene was significantly downregulated in HGCIN compared to normal cervix ( $p=.012$ ), depicted in Fig. 2.

\section{Discussion}

Cervical carcinogenesis is the neoplastic transformation from normal epithelial cells to cervical intraepithelial neoplasia (CIN), and further into invasive cancer. Histologically, LGCIN or CIN 1, consists of dysplastic cells in the lower one-third of the cervical epithelium, while in HGCIN, comprising CIN 2 and CIN 3, at least two-thirds of the thickness of the epithelium is affected (Holowaty et al., 1999). The dysplastic cells hyper-proliferate within the cervical epithelium before turning cancerous and invading into the deeper subepithelial tissues with the potential to metastasize. Majority of cervical cancers are associated with human papillomavirus (HPV) infection especially HPV 18 and 16 (Lorincz et al., 2002; Smith et al., 2007). The viral oncoproteins E6 and E7, dysregulate the host cell cycle by interacting with tumor suppressor protein, $\mathrm{p} 53$, and retinoblastoma $\mathrm{pRb}$, respectively (Longworth and Laimins, 2004). These interactions cause cells to proliferate, grow, differentiate, and avert apoptosis.

The IGF system which regulates cell growth, differentiation, and apoptosis, is partly controlled by IGFBP-2. Both have been demonstrated to play a role in cancer. It is still unclear whether IGFBP-2 acts in a tumor suppressor or oncogenic manner in cervical carcinogenesis. This study aimed to investigate the immunohistochemical expression of IGFBP-2 protein in cervical tissues through the process of carcinogenesis. To further understand the role of IGFBP-2 and IGF-related cellular mechanisms, transcriptomics profiling of the relevant genes were performed on tissues from normal cervix, HPV-associated LGCIN, HGCIN, and SCC.

In tumors, IGFBP-2 expression has been demonstrated to be either up or downregulated depending on the cancer type. In our study, immunohistochemical staining of 71 cases in tissue microarrays showed that IGFBP-2 protein is ubiquitous and present across a wide range of cervical cells, from normal to dysplastic (CIN) and neoplastic (SCC) cells. IGFBP-2 expression was significantly higher (histoscore $\geq 4 / 12$ ) in SCC compared to normal cervix $(p=.002)$, but not significantly different from CIN. Notwithstanding the limited numbers of normal, LGCIN and HGCIN cases, the results support the role of IGFBP-2 as a promoter in the neoplastic transformation of cervical cells. Our results contradict another study in which IGFBP-2 tissue expression was reduced in HPV16-associated CIN 3 and invasive cancer, compared to CIN 1 (Pickard et al., 2015). The HPV status of cases in our tissue microarrays was unknown.

The localization of IGFBP-2 in the cytoplasm and membranes of squamous epithelial cells in our study alludes to its possible interaction with the extracellular matrix components, cell surface proteoglycans and integrin receptors on the cell membrane. IGFBP-2 may be imported into the cell cytoplasm where it can interact with other transcription factors, cytoplasm-nuclear transporters and cell cycle inhibitors such as p21 and Pim-1 associated protein-1 (PAP-1)-associated protein-1 (PAPA-1) (Yau et al., 2015). The nuclear import of IGFBP-2 promotes transcriptional activation of specific genes (Azar et al., 2014; Russo et al., 2015; Yau et al., 2015). Though nuclear localization has been

Table 2

IGFBP-2 histoscore in normal cervix, LGCIN, HGCIN and SCC. The histoscore in individual histological groups was compared to normal cervix.

\begin{tabular}{|c|c|c|c|c|c|c|}
\hline \multirow{2}{*}{$\begin{array}{l}\text { Histological } \\
\text { group }\end{array}$} & \multicolumn{3}{|c|}{ IGFBP-2 histoscore } & \multirow{2}{*}{$\begin{array}{l}\text { Total } \\
n(\%)\end{array}$} & \multirow{2}{*}{$\begin{array}{l}\text { Comparison between all histological groups } \\
p \text { value }\end{array}$} & \multirow{2}{*}{$\begin{array}{l}\text { Histological group vs normal } \\
p \text { value }\end{array}$} \\
\hline & $\begin{array}{l}\text { Negative } \\
n(\%)\end{array}$ & $\begin{array}{l}\text { Low } \\
n(\%)\end{array}$ & $\begin{array}{l}\text { High } \\
n(\%)\end{array}$ & & & \\
\hline Normal & $0(0.0)$ & $7(77.8)$ & $2(22.2)$ & $9(100)$ & & \\
\hline LGCIN & $1(10.0)$ & $3(30.0)$ & $6(60.0)$ & $10(100)$ & & 0.102 \\
\hline HGCIN & $1(10.0)$ & $5(50.0)$ & $4(40.0)$ & $10(100)$ & 0.013 & 0.377 \\
\hline SCC & $1(2.4)$ & $8(19.1)$ & $33(78.6)$ & $42(100)$ & & 0.002 \\
\hline Total & $3(4.2)$ & $23(32.4)$ & $45(63.4)$ & $71(100)$ & & \\
\hline
\end{tabular}

Chi-square test, statistical significance set at $p$ value $<.05$.

Abbreviations: LGCIN, low grade cervical intraepithelial neoplasia; HGCIN, high grade cervical intraepithelial neoplasia; SCC, squamous cell carcinoma. 

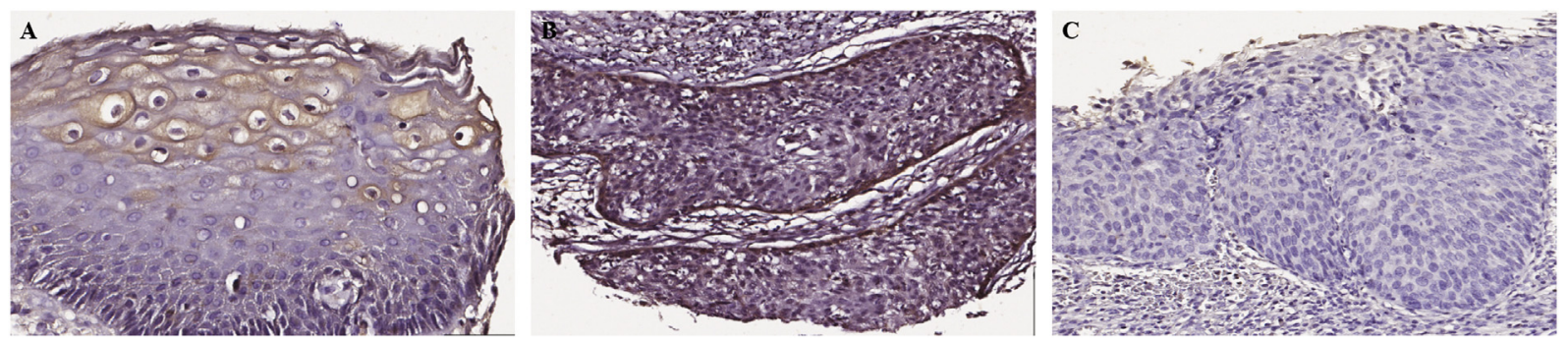

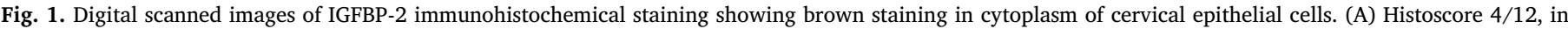

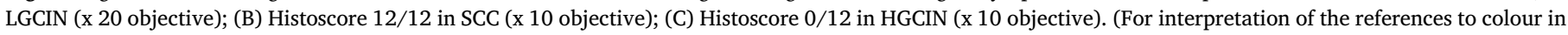
this figure legend, the reader is referred to the web version of this article.)

Abbreviations: LGCIN, low grade cervical intraepithelial neoplasia; HGCIN, high grade cervical intraepithelial neoplasia; SCC, squamous cell carcinoma

reported in some cancer cell lines (Azar et al., 2014), our study did not exhibit nuclear staining of cervical cells, suggesting this mechanism was not significantly involved in cervical carcinogenesis. This was in concordance with another report demonstrating immunohistochemical cytoplasmic staining of cervical epithelium (Pickard et al., 2015). It was interesting to note that the stromal inflammatory cells were positively stained in our study, reflecting the presence of IGFBP-2 in many types of tissue including the immune system.

IGFBP-2 was upregulated in SCC at gene and protein levels in our study. Under these circumstances, IGFBP-2 would competitively bind to the ligand IGF-1 to limit the interaction with IGF-1R, hence reducing IGF-mitogenic activity. Concomitantly, IGF-1 and IGF-1R genes were downregulated, with IGF-1 being significantly downregulated in cervical cancer compared to normal cervix $(p=.031)$. This suggests that the IGFBP-2-IGF dependent pathway did not play a crucial role in cervical cancer development. This adds to the contradictory results obtained by other researchers, clearly summarized and discussed by Durzyńska (Durzyńska, 2014). While most studies support increased IGFs levels in serum or tissue in cervical cancer progression (Lee et al., 2010; Wu et al., 2003), others observed reductions of IGFs or IGFRs (Schaffer et al., 2007; Serrano et al., 2007; Serrano et al., 2006). An in vivo study on CIN lesions showed that IGFBP-2 expression was reduced as the disease progressed from CIN 1 to CIN 3, leading to activation of the IGF/IGFR pathways (Pickard et al., 2015). This contrasted with our findings of increased IGFBP-2 gene expression in HGCIN and SCC.

In our study, the transcriptome profile of IGFBP-2 was comparable to TP53 expression. Both genes were upregulated in HGCIN and SCC groups. In HPV-associated cervical cancer, it has been established that E6 binds to p53, causing inhibition of apoptosis (Longworth and Laimins, 2004), a key factor allowing DNA-damaged cells to proliferate and transform into cancerous cells. IGFBP-2 has been proposed to be regulated by $\mathrm{p} 53$, which in turn inhibits IGF-1 signaling (Grimberg et al., 2006), as observed in our study.

PTEN is a tumor suppressor gene which is frequently mutated in a variety of human cancers and is associated with tumor progression ( $\mathrm{Li}$ et al., 1997; Mehrian-Shai et al., 2007). At the cellular level, the expression of IGFBP-2 is influenced by the functional status of PTEN (Dean et al., 2014; Levitt et al., 2005; Mehrian-Shai et al., 2007; Perks et al., 2007). Loss of function of PTEN in cancers is often associated with high levels of IGFBP-2 expression. Studies conducted on breast and glioma cell lines support the inverse relationship between IGFBP-2 and PTEN (Fukushima and Kataoka, 2007; Levitt et al., 2005; Perks et al., 2007). Loss of PTEN in triple negative breast cancer was associated with increased immunohistochemical expression of IGFBP-2 (Dean et al., 2014). Our findings concur, exhibiting an inverse relationship between PTEN and IGFBP-2 gene expressions in HGCIN and SCC groups. PTEN gene was significantly downregulated in HGCIN compared to normal cervix $(p=.012)$, which may offer a growth advantage in the progression from a precancerous state to cancer. IGFBP-2 appears to exert an oncogenic potential, which is independent of IGF. This signaling may be mediated via integrin receptors, leading to inhibition of PTEN, which in turn activates the AKT pathway, contributing to cell survival and proliferation (Kumar, 1998; Uzoh et al., 2011).

Binding of IGF-1R to ligand stimulates intracellular activities that regulate cell survival and proliferation. The PI3-AKT pathway plays a critical role in the activation of anti-apoptotic factor, MDM2 and proinflammatory factor, NF- KB (Mayo and Donner, 2001; Vivanco and Sawyers, 2002). Phosphorylation of MDM2 by AKT promotes its nuclear translocation and binding to p53 tumor suppressor gene leading to degradation of p53 (Mayo and Donner, 2001; Moll and Petrenko, 2003; Vivanco and Sawyers, 2002). Similarly, AKT causes phosphorylation followed by translocation of NF- кB from the cytoplasm into the

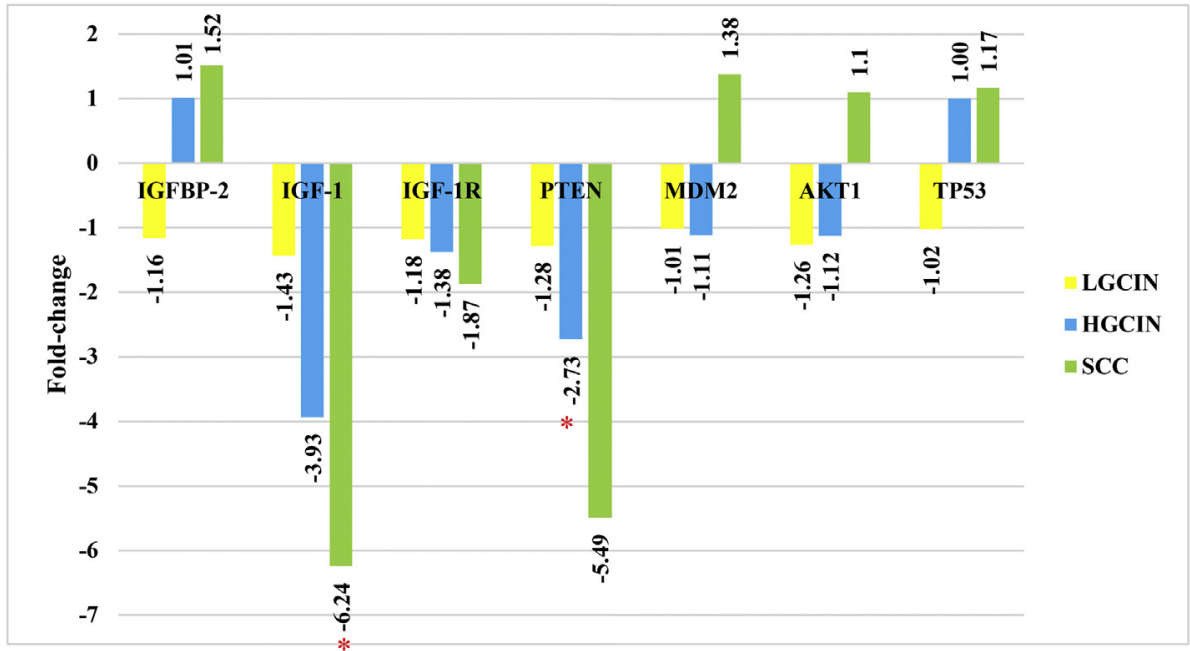

Fig. 2. Transcriptomic profiles of IGFBP-2, IGF-1, IGF-1R, PTEN, MDM2 and AKT1 in LGCIN, HGCIN and SCC obtained from Human Transcriptome Array. Bars represent the fold change in each histological group compared to normal cervix. One-way ANOVA was done using the array software. * represents $p$ value $<.05$

Abbreviations: LGCIN, low grade cervical intraepithelial neoplasia; HGCIN high grade cervical intraepithelial neoplasia; SCC, squamous cell carcinoma; AKT, protein kinase B; IGFBP-2, insulin-like growth factor binding protein; IGF-1, insulin-like growth factor 1; IGF-1R, insulin-like growth factor 1 receptor; MDM2, murine double minute 2; NF- кB, nuclear factor of kappa B; PI3-K, phosphatidylinositol 3-kinase; PTEN, phosphatase and tensin homolog 


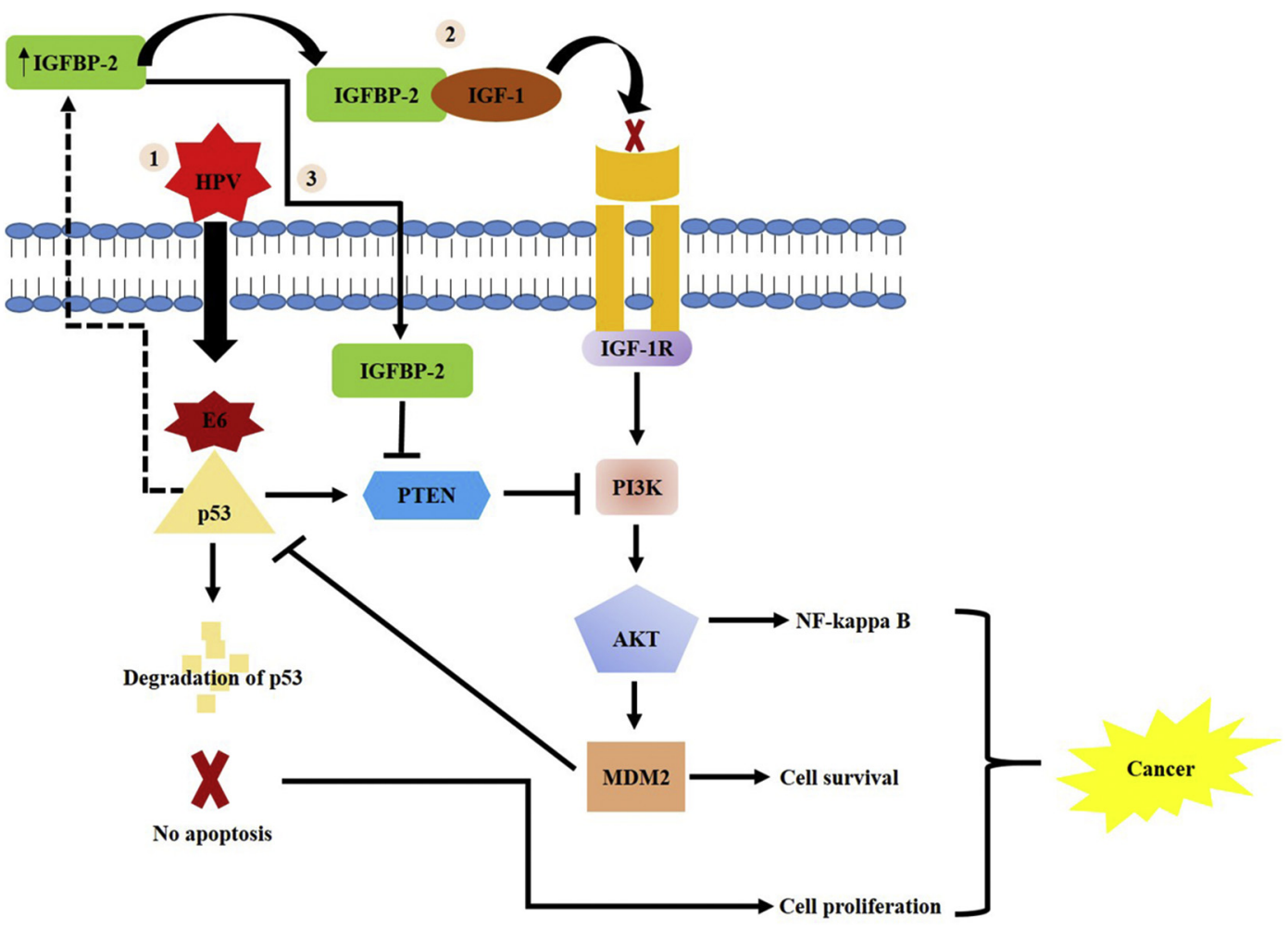

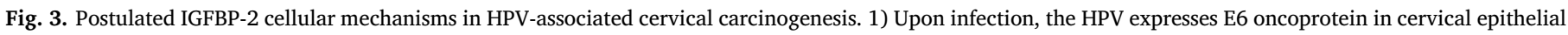

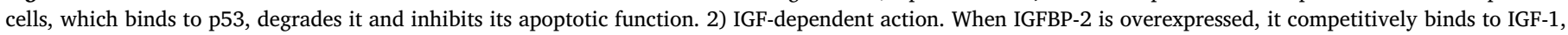

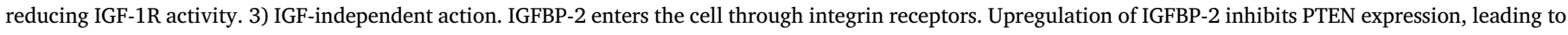
activation of PI3K-AKT pathway, NF-KB, and MDM2, promoting cell survival and cell proliferation.

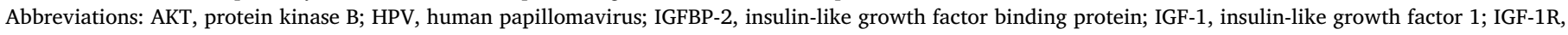

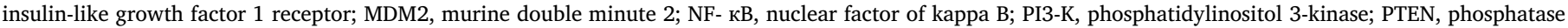
and tensin homolog

nucleus, allowing activation of target genes (Vivanco and Sawyers, 2002). Hyperactivation of the PI3K-AKT pathway in tumors has been shown to promote cancer cell proliferation, survival, and invasion (Tang et al., 2018). The results of our study showed downregulation of AKT1 and MDM2 in LGCIN and HGCIN, while upregulated in SCC, suggesting cancer cells acquire cell survival and anti-apoptotic properties.

In postulating the IGF system mechanisms involved in cervical cancer development, it is important to appreciate the different stages of tumor progression (Fig. 3). In the initial stage, the integration of HPV oncoproteins into the host cells causes deregulation of the cell cycle, leading to cell proliferation. During this period, the virus attempts to evade the host immune system (Gius et al., 2007). In our study, IGFBP-2 was downregulated in LGCIN, while the various genes involved in the IGF system showed one to two-fold change difference compared to normal cervix. The IGF signaling pathway did not appear to play a crucial role during this phase. Subsequently, hyper-proliferation and anti-apoptotic mechanisms become crucial in transforming HGCIN into SCC (Niu et al., 2017). Our results exhibited an upregulation of IGFBP-2 associated with downregulation of IGF-1, IGF-1R and PTEN genes during these stages, suggesting the role of IGF-independent mechanisms in promoting cell proliferation. In SCC, IGFBP-2 inhibits PTEN, thus activating the AKT and MDM2 signaling pathways, and promoting cell proliferation and cell survival. The membrane localization of IGFBP-2 protein in CIN and cancer cells implies that it may bind to cell surface proteoglycans or integrin receptors to indirectly promote oncogenesis via IGF-independent actions. Additionally, cytoplasmic IGFBP-2 may trigger other proteins encouraging carcinomatous change. Further research is warranted to identify the specific cellular mechanisms involved.

\section{Conclusion}

We postulate that IGFBP-2 plays an oncogenic role in cervical cancer through the IGF-independent mechanism.

\section{Funding source}

The work was funded by Bridging Research Grant (Grant No. 304/ CIPPM/6316290) and Research University Grant (Grant No. 1001/ CIPPM/812116) from Universiti Sains Malaysia.

\section{Declaration of Competing Interest}

The authors have no competing interests.

\section{Acknowledgement}

We thank Matrix Optics (M) Sdn. Bhd for their support in the digital scanning of tissue microarrays.

\section{References}

Ambrosini-Spaltro, A., et al., 2011. IGFBP2 as an immunohistochemical marker for prostatic adenocarcinoma. Appl. Immunohistochem. Mol. Morphol. 19, 318-328.

Azar, W., et al., 2014. IGFBP-2 nuclear translocation is mediated by a functional NLS sequence and is essential for its pro-tumorigenic actions in cancer cells. Oncogene 33, 578-588.

Dean, S.J., et al., 2014. Loss of PTEN expression is associated with IGFBP2 expression, younger age, and late stage in triple-negative breast cancer. Am. J. Clin. Pathol. 141, 323-333.

Durzyńska, J., 2014. IGF axis and other factors in HPV-related and HPV-unrelated carcinogenesis. Oncol. Rep. 32, 2295-2306. 
Elmlinger, M.W., et al., 2001. In vivo expression of insulin-like growth factor-binding protein-2 in human gliomas increases with the tumor grade. Endocrinology 142, 1652-1658.

Firth, S.M., Baxter, R.C., 2002. Cellular actions of the insulin-like growth factor binding proteins. Endocr. Rev. 23, 824-854.

Flyvbjerg, A., et al., 1997. Elevated serum insulin-like growth factor-binding protein 2 (IGFBP-2) and decreased IGFBP-3 in epithelial ovarian cancer: correlation with cancer antigen 125 and tumor-associated trypsin inhibitor. J. Clin. Endocrinol. Metab. 82, 2308-2313.

Fukushima, T., Kataoka, H., 2007. Roles of insulin-like growth factor binding protein-2 (IGFBP-2) in glioblastoma. Anticancer Res. 27, 3685-3692.

Gius, D., et al., 2007. Profiling microdissected epithelium and stroma to model genomic signatures for cervical carcinogenesis accommodating for covariates. Cancer Res. 67, 7113-7123.

Grimberg, A., et al., 2006. Insulin-like growth factor binding protein-2 is a novel mediator of p53 inhibition of insulin-like growth factor signaling. Cancer Biol. Ther. 5, 1408-1414.

Guo, C., et al., 2013. Insulin-like growth factor binding protein-2 level is increased in blood of lung cancer patients and associated with poor survival. PLoS One 8, e74973.

Ho, J.P., Baxter, R.C., 1997. Insulin-like growth factor-binding protein-2 in patients with prostate carcinoma and benign prostatic hyperplasia. Clin. Endocrinol. 46, 333-342.

Hoeflich, A., et al., 2001. Insulin-like growth factor-binding protein 2 in tumorigenesis: protector or promoter? Cancer Res. 61, 8601-8610.

Holowaty, P., et al., 1999. Natural history of dysplasia of the uterine cervix. J. Natl. Cancer Inst. 91, 252-258.

Janku, F., et al., 2018. Targeting the PI3K pathway in cancer: are we making headway? Nat. Rev, Clin. Oncol. 15, 273-291.

Jiang, R., et al., 2006. Pathway alterations during glioma progression revealed by reverse phase protein lysate arrays. Proteomics. 6, 2964-2971.

Kumar, C.C., 1998. Signaling by integrin receptors. Oncogene. 17, 1365-1373.

Lee, S.W., et al., 2010. Plasma levels of insulin-like growth factor-1 and insulin-like growth factor binding protein-3 in women with cervical neoplasia. J. Gynecol. Oncol. 21, 174-180.

Levitt, R.J., et al., 2005. PTEN-induction in U251 glioma cells decreases the expression of insulin-like growth factor binding protein-2. Biochem. Biophys. Res. Commun. 336, 1056-1061.

Li, J., et al., 1997. PTEN, a putative protein tyrosine phosphatase gene mutated in human brain, breast, and prostate cancer. Science. 275, 1943-1947.

Liou, J.-M., et al., 2010. Plasma insulin-like growth factor-binding protein-2 levels as diagnostic and prognostic biomarker of colorectal cancer. J. Clin. Endocrinol. Metab. $95,1717-1725$.

Longworth, M.S., Laimins, L.A., 2004. Pathogenesis of human papillomaviruses in differentiating epithelia. Microbiol. Mol. Biol. Rev. 68, 362-372.

Lorincz, A., N, M., Meijer, C.J., Shah, K.V., 2002. The causal relation between human papillomavirus and cervical cancer. J. Clin. Pathol. 55, 244-265.

Mayo, L.D., Donner, D.B., 2001. A phosphatidylinositol 3-kinase/Akt pathway promotes translocation of Mdm2 from the cytoplasm to the nucleus. Proc. Natl. Acad. Sci. 98, 11598-11603.

Mehrian-Shai, R., et al., 2007. Insulin growth factor-binding protein 2 is a candidate biomarker for PTEN status and PI3K/Akt pathway activation in glioblastoma and prostate cancer. Proc. Natl. Acad. Sci. 104, 5563-5568.

Moll, U.M., Petrenko, O., 2003. The MDM2-p53 interaction. Mol. Cancer Res. 1, $1001-1008$

Momand, J., et al., 1998. The MDM2 gene amplification database. Nucleic Acids Res. 26, 3453-3459.

Nag, S., et al., 2013. The MDM2-p53 pathway revisited. J. Biomed. Res. 27, 254-271.

Niu, G., et al., 2017. Systematic identification of key genes and pathways in the development of invasive cervical cancer. Gene. 618, 28-41.

Perks, C., et al., 2007. IGF-II and IGFBP-2 differentially regulate PTEN in human breast cancer cells. Oncogene. 26, 5966-5972.

Pickard, A., McCance, D.J., 2015. IGF-binding protein 2-oncogene or tumor suppressor? Front. Endocrinol. 6, 1-8.

Pickard, A., et al., 2015. HPV16 down-regulates the insulin-like growth factor binding protein 2 to promote epithelial invasion in organotypic cultures. PLoS Pathog. 11, e1004988.

Pollak, M.N., et al., 2004. Insulin-like growth factors and neoplasia. Nat. Rev. Cancer 4, 505.

Renehan, A.G., et al., 2000. Elevated serum insulin-like growth factor (IGF)-II and IGF binding protein-2 in patients with colorectal cancer. Br. J. Cancer 83, 1344-1350.

Romashkova, J.A., Makarov, S.S., 1999. NF-kB is a target of AKT in anti-apoptotic PDGF signalling. Nature. 401, 86-90.

Rosenzweig, S.A., 2004. What's new in the IGF-binding proteins? Growth Hormon. IGF Res. 14, 329-336.

Russo, V., et al., 2015. IGFBP-2: the dark horse in metabolism and cancer. Cytokine Growth Factor Rev. 26, 329-346.

Sayer, R.A., et al., 2005. High insulin-like growth factor-2 (IGF-2) gene expression is an independent predictor of poor survival for patients with advanced stage serous epithelial ovarian cancer. Gynecol. Oncol. 96, 355-361.

Schaffer, A., et al., 2007. Insulin-like growth factor-I and risk of high-grade cervical intraepithelial neoplasia. Cancer Epidemiology and Prevention Biomarkers. 16, $716-722$.

Schutt, B., et al., 2004. Integrin-mediated action of insulin-like growth factor binding protein-2 in tumor cells. J. Mol. Endocrinol. 32, 859-868.

Serrano, M.L., et al., 2006. Serum levels of insulin-like growth factor-I and-II and insulinlike growth factor binding protein 3 in women with squamous intraepithelial lesions and cervical cancer. Biomedica. 26, 258-268.

Serrano, M.-L., et al., 2007. Insulin-like growth factor system gene expression in cervical scrapes from women with squamous intraepithelial lesions and cervical cancer. Growth Hormon. IGF Res. 17, 492-499.

Smith, J.S., et al., 2007. Human papillomavirus type distribution in invasive cervical cancer and high-grade cervical lesions: a meta-analysis update. Int. J. Cancer 121 , 621-632.

Tang, F., et al., 2018. PKB/Akt-dependent regulation of inflammation in cancer. In: Seminars in Cancer Biology. Vol. 48. Elsevier, pp. 62-69.

Uhlen, M., et al., 2010. Towards a knowledge-based human protein atlas. Nat. Biotechnol $28,1248-1250$.

Uzoh, C., et al., 2011. Insulin-like growth factor-binding protein-2 promotes prostate cancer cell growth via IGF-dependent or-independent mechanisms and reduces the efficacy of docetaxel. Br. J. Cancer 104, 1587-1593.

Vivanco, I., Sawyers, C.L., 2002. The phosphatidylinositol 3-kinase-AKT pathway in human cancer. Nat. Rev. Cancer 2, 489-501.

Wang, H., et al., 2006. Insulin-like growth factor-binding protein 2 and 5 are differentially regulated in ovarian cancer of different histologic types. Mod. Pathol. 19, 1149.

Weroha, S.J., Haluska, P., 2012. The insulin-like growth factor system in cancer. Endocrinol. Metab. Clin. N. Am. 41, 335-350.

Wu, X., et al., 2003. Serum levels of insulin-like growth factor I and risk of squamous intraepithelial lesions of the cervix. Clin. Cancer Res. 9, 3356-3361.

Yau, S.W., et al., 2015. IGFBP-2-taking the lead in growth, metabolism and cancer. J. Cell. Commun. Signal. 9, 125-142.

Yazawa, T., et al., 2009. Neuroendocrine cancer-specific up-regulating mechanism of insulin-like growth factor binding protein-2 in small cell lung cancer. Am. J. Pathol 175, 976-987.

Zhi, W., et al., 2014. Twelve serum proteins progressively increase with disease stage in squamous cell cervical cancer patients. International Journal of Gynecologic Cancer. 24, 1085-1092.

Zhou, Q., et al., 2012. Development of IGF signaling antibody arrays for the identification of hepatocellular carcinoma biomarkers. PLoS One 7, e46851. 